\title{
Intracranial silicone oil migration with encephalopathy: a rare case report
}

\author{
Juvaina Puthiyakam ${ }^{* *}$ D, Najma Nalakath', Devarajan Ezhuthullathil ${ }^{1}$, Faiz Riyaz Arakkal², \\ Saanida Monthampally ${ }^{1}$ and Athira Prasad
}

\begin{abstract}
Background: Intraocular silicone oil ( $\mathrm{SiO}$ ) tamponade is a popular modality of treatment of rhegmatogenous retinal detachment. It can, however, rarely lead to an incompletely understood phenomenon known as intracranial migration of SiO. Though usually benign, this entity is often misinterpreted as a tumour or haemorrhage.

Case presentation: We present a case report of a patient with acute progressive encephalopathy and intracranial migration of intravitreal silicone. We also put forward the possibility of toxic effects of intracranial SiO on the central nervous system.

Conclusion: Clinicians and radiologists should be aware of the rare complication of intravitreal SiO tamponade. Eliciting the history of intraocular $\mathrm{SiO}$ tamponade and identifying the key imaging features on ultrasonography of the orbit, CT of the head, and MRI of the brain will help in avoiding unnecessary interventions or delays in the management of the patients from misdiagnosing it as haemorrhage or tumour.
\end{abstract}

Keywords: Silicone oil, Intraocular, Intraventricular, Intracranial migration, Encephalopathy, Case report

\section{Background}

Intraocular silicone oil $(\mathrm{SiO})$ tamponade is a popular modality of treatment of rhegmatogenous retinal detachment. It can, however, rarely lead to an incompletely understood phenomenon known as intracranial migration of silicone oil. Though usually benign, this entity is often misinterpreted as a tumour or haemorrhage.

\section{Case presentation}

A 50-year-old ex-alcoholic male patient, with a past medical history of type 2 diabetes mellitus of 30 years duration and one episode of cerebrovascular accident (facial deviation with slurring of speech) 4 months back, presented to the emergency department with complaints of abnormal behaviour and irrelevant talk of 1-week duration followed by 1 day of decreased responsiveness. $\mathrm{He}$

\footnotetext{
*Correspondence: juvaina.faiz@gmail.com

${ }^{1}$ Department of Radiodiagnosis, Government Medical College,

Kozhikode, Kerala 673008, India

Full list of author information is available at the end of the article
}

had a history of progressive visual impairment in both eyes for the past 4 years, from bilateral proliferative diabetic retinopathy (PDR) and bilateral immature senile cataracts (IMSC). He underwent laser surgery in the right eye for PDR, followed by the development of vitreous haemorrhage and retinal detachment (RD) in the postoperative period, which was treated by vitrectomy and silicone oil ( $\mathrm{SiO}$ ) implantation. At the time of admission to our hospital, he was alert, mute, and showed distractibility, poor understanding, reduced attention, and bizarre behaviour. Grasping, choreiform movements in the lower limbs, and myoclonic movements of the trunk, without gait disturbance, were present. Later, he became drowsy; there were no motor or sensory deficits. Ocular examination revealed blind eyes with cataractous lenses bilaterally (Table 1).

Given the acute progressive encephalopathy-like clinical presentation, the initial considerations were metabolic, toxic, and inflammatory causes. However, an emergency NCCT head evaluation was done to rule out the possibility of a cerebrovascular accident (CVA). 
Table 1 Summary of the case

\begin{tabular}{|c|c|c|}
\hline Patient (age, gender) & 1 & 50-year old, male \\
\hline Final diagnosis & 2 & Intracranial silicone oil migration with silicone oil-induced encephalopathy \\
\hline Symptoms & 3 & Abnormal behaviour, decreased responsiveness \\
\hline Past history & 4 & Type 2 diabetes, proliferative diabetic retinopathy (PDR) \\
\hline Past surgical history & 5 & $\begin{array}{l}\text { Laser for PDR in right eye } \\
\text { Vitrectomy and silicone oil implantation for vitreal haemorrhage and retinal detachment }\end{array}$ \\
\hline Clinical procedure & 6 & Plain CT head, USG orbits, MRI Brain \\
\hline Specialty & 7 & Radiology \\
\hline Objective & 8 & To show one of the rare complication of the pathology \\
\hline Background & 9 & Intracranial migration of silicon oil can cause encephalopathy \\
\hline Case report & 10 & Intracranial silicone oil migration with encephalopathy: A rare case report \\
\hline Conclusions & 11 & $\begin{array}{l}\text { History of intraocular SiO implantation should be elicited in any case of non-dependant } \\
\text { intracranial hyperdense lesions in CT. MRI of the brain will help to differentiate it from } \\
\text { haemorrhage or tumour }\end{array}$ \\
\hline MeSH Keywords & 12 & Silicone oil, intraocular, intraventricular, intracranial migration, encephalopathy, case report \\
\hline
\end{tabular}

It showed multiple chronic infarcts involving centrum semi ovale, corona radiata, thalamus, and gangliocapsular region bilaterally. Besides, two well-defined globular hyperdense lesions of different sizes (measuring $11 \times 10 \mathrm{~mm}$ and $4 \times 5 \mathrm{~mm}$ ) and average HU 80 were noted in a non-dependent location within the frontal horns of bilateral lateral ventricles (Figs. 1, 3). Multiple (3 in no.) similar hyperdense globular structures were also noted in the posterior chamber of the right eye with average HU 95 (Fig. 2). The right optic nerve, right half of the optic chiasm, and the right optic tract appeared hyperdense, with an average $\mathrm{HU}$ of 60-70. There was evidence of bilateral retinal detachment (subretinal hyperdensity with characteristic indentation at the optic disc) and bilateral cataracts. Multiple small scleral calcifications (average HU 150) were noted in the posterior aspect of the left globe. Features of small vessel ischaemic disease and age-related brain atrophy were also noted (Fig. 3).

His previous MRI of the brain which was done 4 months back (when he presented with CVA) performed from a private scanning centre was available. It showed acute infarct in the left corona radiata and narrowing of P3 and P4 segments of bilateral posterior cerebral arteries. Globular T1 and T2 hyperintense lesions (relative to vitreous and CSF) were noted in the frontal horns of bilateral lateral ventricles and posterior chamber of right eye corresponding to our CT images, as well as an additional globule was noted in the right temporal horn (the size of the lesion in right frontal horn appeared larger in our CT study, possibly due to migration of the globule from the right temporal horn). At that point of time, as CT correlation showed hyperdense lesions, they had considered the diagnosis of focal haemorrhages/ metastatic lesions within the ventricles in the background of eye disease: hyperintensities in the right globe-likely vitreous haemorrhage/ mass lesion; \& altered signals in the left globe-likely old retinal detachment/mass lesion.

Meanwhile, we were able to elicit the history of right eye surgery. Thus, considering the non-dependent nature of the intraventricular hyperdensities, their similarities with intraocular contents in the right eye (status postsilicone oil tamponade), and the demonstration of the change in position of the lesions (compared to previous MRI), we were prompted to consider a diagnosis of intraventricular silicon oil migration.

Even then, the patient's clinical condition could not be explained with the present imaging findings. Subsequently, an MR Imaging of the brain was done (about 10 days later than the $\mathrm{CT}$ ) which revealed multiple small acute infarcts involving corona radiata, centrum semi ovale, posterior limb of the internal capsule, cerebellar hemisphere, and corpus callosum on the left side. The intraventricular globular lesions were now found to occupy the third ventricle and temporal horn of the right lateral ventricle (Fig. 4) confirming the mobile nature of the lesions. Demonstration of the typical chemical shift artefact at the interface of the oil globule and CSF was confirmatory of intraventricular $\mathrm{SiO}$ migration.

Retrospective ultrasound evaluation of both globes (Figs. 5, 6) showed findings consistent with bilateral RD, bilateral IMSC (immature senile cataracts), and intravitreal silicone oil in the right eye (Fig. 5). On ultrasound, $\mathrm{SiO}$ appeared anechoic, with hyperreflective dots within it (due to emulsified $\mathrm{SiO}$ droplets) and arc-like echogenic bands at its interface with the vitreous. Pseudo-elongation of the optical axis was also noted. Further ophthalmological evaluation also revealed secondary glaucoma due to emulsified $\mathrm{SiO}$ in the right eye.

Meanwhile, the patient was evaluated for the alternate causes of acute encephalopathy. However, the 


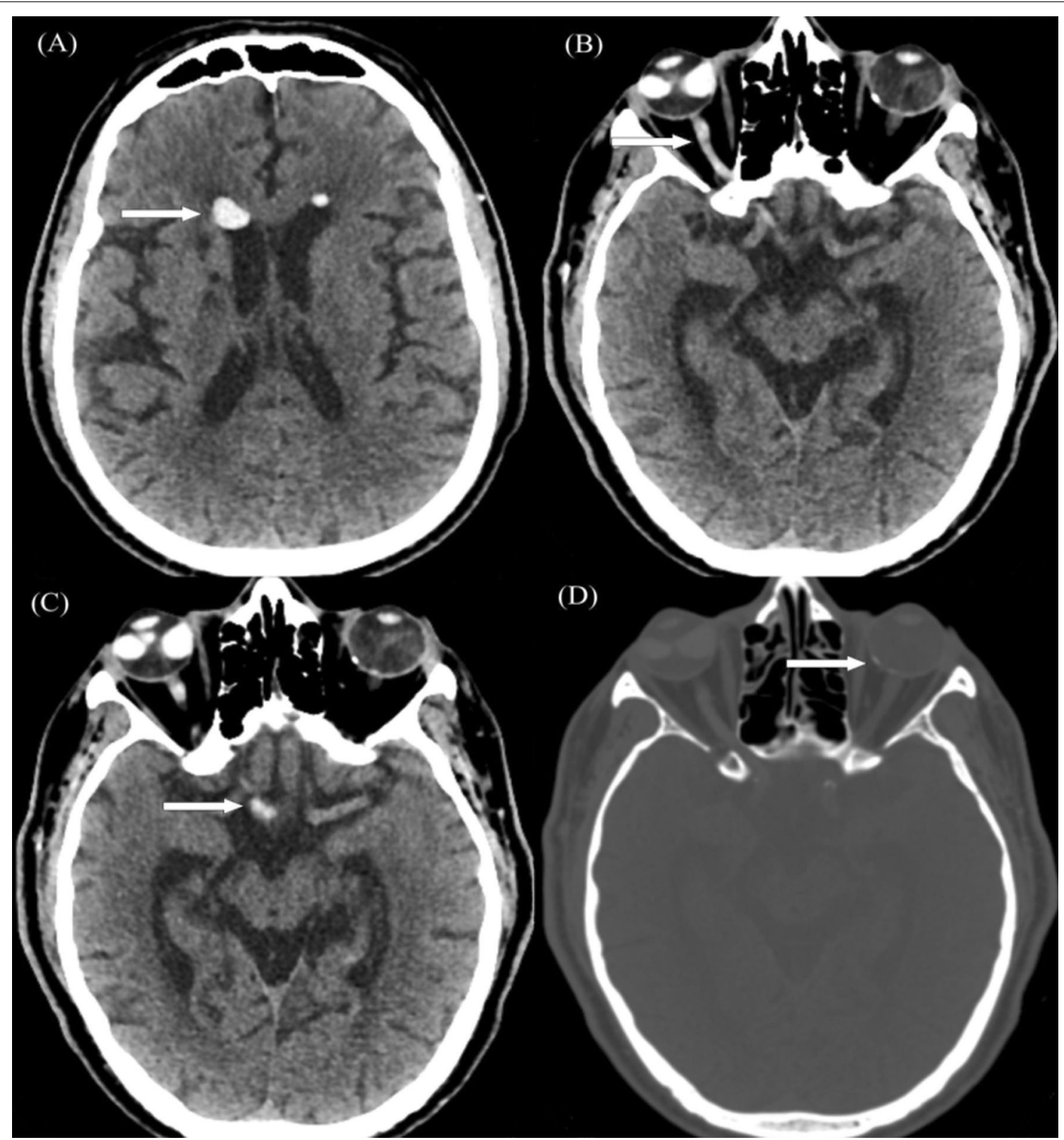

Fig. 1 NCCT axial sections of the head show multiple hyperdense foci (arrows) - A Globular hyperdense lesions (average HU of 80) in frontal horns of bilateral lateral ventricles. Also, note the small hypodensities in the right gangliocapsular region suggestive of chronic lacunar infarcts. B Hyperdense globules within the vitreous chamber of the right globe and linear hyperdensity along the right optic nerve. C The linear hyperdensity is noted extending up to the right side of the optic chiasma. D Bone window shows small scleral calcifications in the left globe

haematologic, metabolic, autoimmune, and paraneoplastic workups came out unremarkable. So, we were prompted to consider the remote possibility of a toxic effect of silicone oil on the CNS to account for his symptoms. Thus, a final diagnosis of intracranial silicone oil migration with silicone oil-induced encephalopathy was considered after excluding other causes.

\section{Discussion}

Retinal detachment (RD) is an ocular disease where the neurosensory layer of the retina gets separated from the retinal pigment epithelium. Depending on the mechanism, RD can be classified as either rhegmatogenous, tractional, or exudative RD. In rhegmatogenous RD, a full-thickness retinal tear develops, followed by the entry 

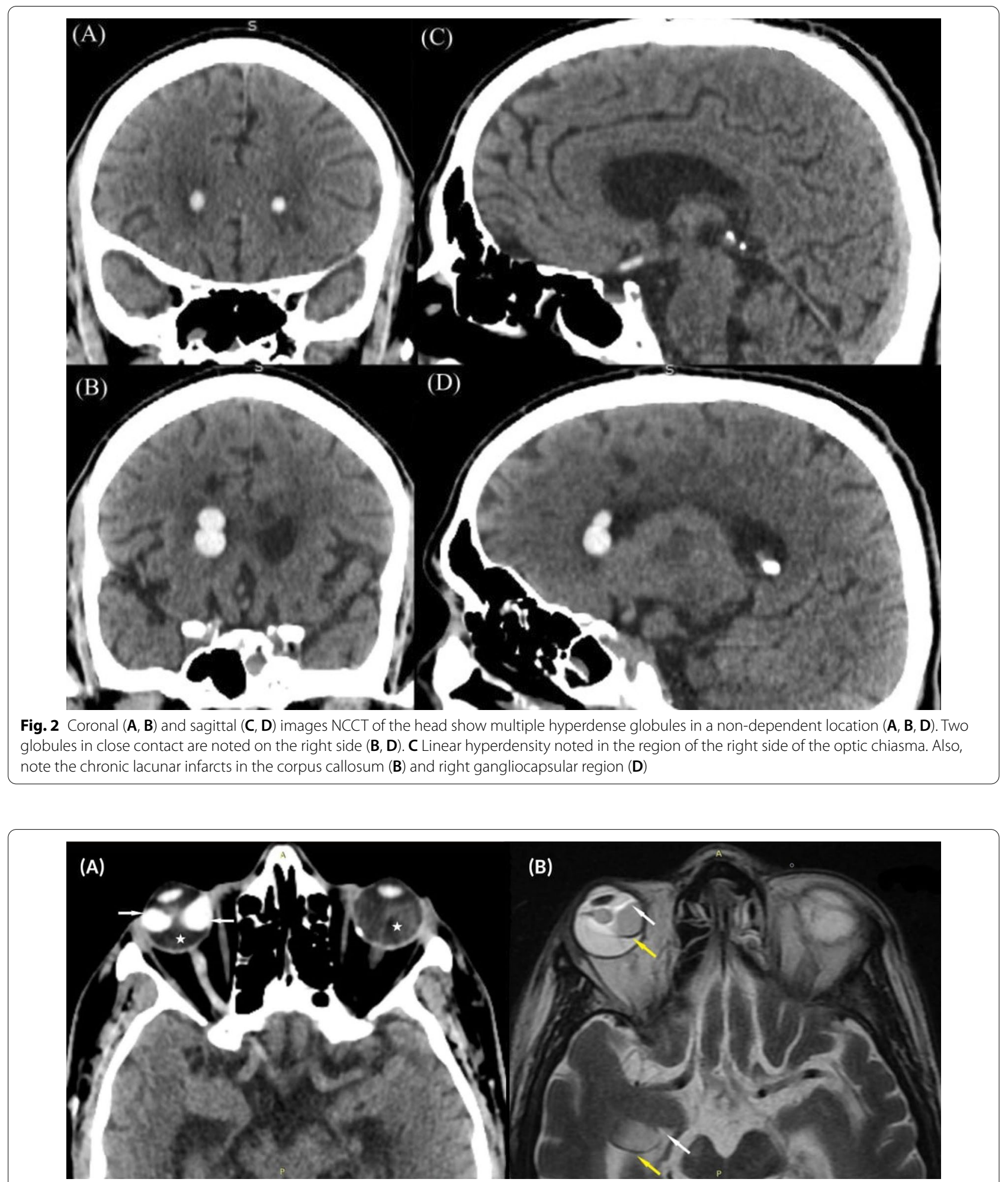

Fig. 3 A Axial NCCT image of bilateral orbits shows silicone oil globules in the right vitreous chamber (arrows) and silicone oil tracking along the right optic nerve sheath. Also, note the partially treated retinal detachment on the right side and an untreated total retinal detachment on the left side (asterisks). B Axial T2W MR image of the right globe shows chemical shift artefact at the interface of the silicone oil with the vitreous chamber, seen as thin curvilinear hyperintense band anteriorly (white arrows) and thin hypointense band posteriorly (yellow arrows). Intraventricular silicone oil globule located within the right temporal horn (which is not seen in the prior CT image, owing to its mobile nature) showing chemical shift artefact 


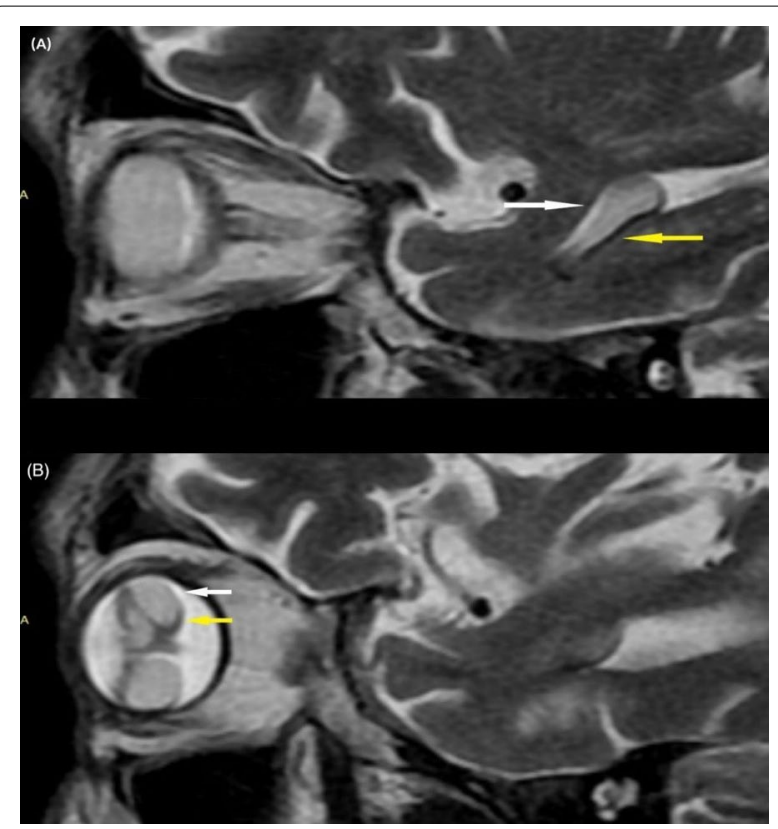

Fig. 4 Sagittal T2W MR images give a better look at the chemical shift artefact produced at the interface by the silicone oil within the temporal horn of the right lateral ventricle $(\mathbf{A})$ and the vitreous chamber of the right globe (B). A thin curvilinear hyperintense band (white arrows) is noted superior to the globule and a thin hypointense band (yellow arrows) is noted inferior to the globule

of liquid vitreous into the subretinal space. The risk factors implicated are ageing, posterior vitreous detachment, severe myopia, previous cataract surgery, and ocular trauma. Tractional RD occurs due to the mechanical pull on the retina, by vitreoretinal traction forces as in conditions such as proliferative diabetic retinopathy and retinopathy of prematurity. Exudative RD, on the other hand, is characterized by the accumulation of subretinal fluid, which is seen with inflammatory conditions like Coats disease or ocular malignancies [1].

The various treatment modalities for RD include scleral buckling, pneumatic retinopexy, laser photocoagulation, vitrectomy, and gas or silicone oil injection. Endotamponade with gases like $\mathrm{SF}_{6}$ and $\mathrm{C}_{3} \mathrm{~F}_{8}$ will lead to spontaneous dissipation of the gas with time. However $\mathrm{SiO}$ is permanent and hence needs removal usually after 3 months, and if required, removal may be delayed up to 18 months [1].

The common complications associated with silicone oil tamponade are band keratopathy, cataract, emulsification of oil followed by secondary glaucoma, fibrous epiretinal and subretinal proliferation, subconjunctival and subretinal migration of oil droplets, etc. [2]. SiO tamponade can also lead to severe optic neuropathy caused by retrolaminar migration of $\mathrm{SiO}$. Further intracranial migration of $\mathrm{SiO}$ into the cerebral ventricles is very rare. It is often incidentally detected on imaging while evaluating non-specific symptoms like headaches, but associated visual problems are common.

Many cases of $\mathrm{SiO}$ migration had been documented since it was first reported in 1994 [3]. Many risk factors and mechanisms of action had been identified. Even then its pathophysiology remains uncertain. Several theories had been put forward by various authors.

An active silicone transport system had been proposed by Papp et al. [4], whereby silicone-filled macrophages actively transport $\mathrm{SiO}$ from the vitreous cavity to the subarachnoid space. The hypothesis was based on a histopathologic study identifying CD68-positive macrophages positive for $\mathrm{SiO}$ at the border of the vacuoles in the optic nerve [4].

A common neurological complication following retrolaminar $\mathrm{SiO}$ migration is severe optic neuropathy leading to visual deficits. The oil may replace up to $40 \%$ of the cross-sectional area of the retrolaminar optic nerve and may penetrate deep into it. Granulomatous inflammatory reactions surrounding retrolaminar silicone oil are found to be responsible for the optic nerve damage in some eyes [5]. We postulate that a similar inflammatory process induced by intracranial $\mathrm{SiO}$ might also lead to an acute progressive encephalopathy-like state, as in this case we have presented.

\section{Diagnostic imaging features}

$\mathrm{SiO}$ has been described as hyperdense on NCCT with density ranging from 50 to $130 \mathrm{HU}$, and hence can be misdiagnosed as intraventricular haemorrhage (IVH) when within the ventricles, and as vitreous haemorrhage $(\mathrm{VH})$ when seen in the vitreous chamber [6].

Some newer studies have shown the potential of CT head evaluation in a prone position for prompt and costeffective diagnosis of intraventricular migration of silicone oil by demonstrating the mobility of the oil [7].

On the imaging of the orbits, intravitreal $\mathrm{SiO}$ was seen as homogenously hyperdense globules within the vitreous chamber in NCCT, with characteristic chemical shift artefact and blooming in SWI on MRI. The ipsilateral optic nerve and optic chiasma showed hyperdense attenuation in CT and blooming in SWI on MRI. An orbital ultrasound evaluation helped in differentiating blood from $\mathrm{SiO}$. The latter appeared as anechoic areas filling the vitreous chamber with arc-like hyperechoic bands at the interface of the oil and vitreous. Multiple tiny hyperreflective dots were also seen within the globules owing to the partial emulsification of the oil. 


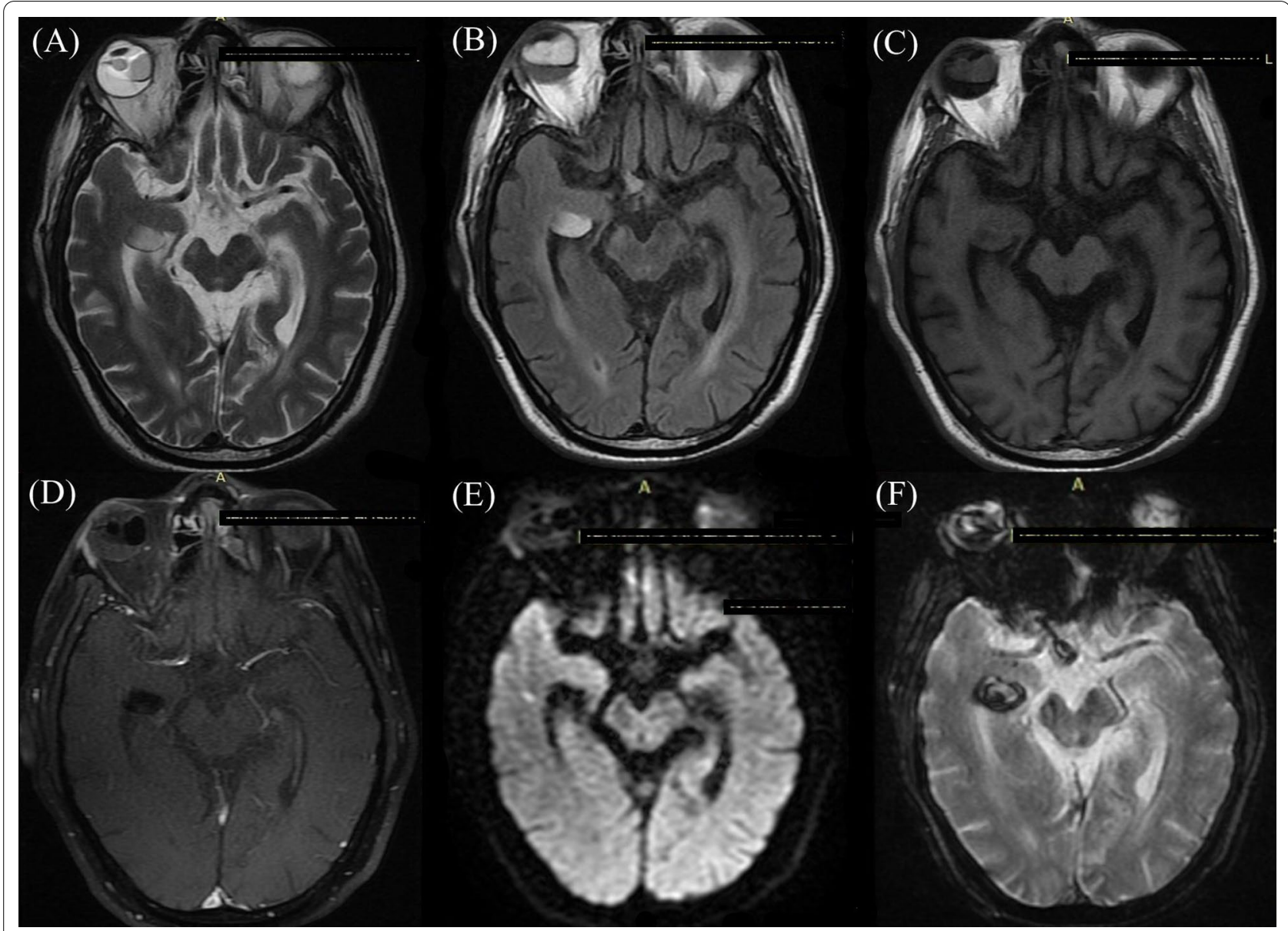

Fig. 5 A Axial T2W MR shows hyperintense silicone oil with characteristic chemical shift artefact within the temporal horn of the right lateral ventricle and the vitreous chamber of the right globe. T2 hyperintensity is also noted in the right side of optic chiasm due to the tracking of silicone oil along the optic nerve. B Axial T2-FLAIR image shows hyperintense silicone oil mimicking intraventricular/intraocular haemorrhage. C Non-contrast Axial T1WI shows hyperintense silicone oil. D Post-contrast Axial T1 Fat Suppressed (T1 FS + C) image shows a lack of enhancement within the globules. E Axial diffusion-weighted image (DWI) shows a lack of diffusion restriction within the globules. F Axial Gradient Recall Echo (GRE) shows susceptibility artefact in the aforementioned areas of T2 hyperintensity

\section{Prognosis and treatment}

$\mathrm{SiO}$ migration is a benign entity, as it is often incidentally detected, although visual problems are commonly associated with it. Few cases of immediate postoperative visual loss associated with perineural subarachnoid silicone oil have been documented [8]. To date, no reports of focal neurological deficits have been documented in cases of intraventricular $\mathrm{SiO}$, to warrant neurosurgical removal of the oil. On the contrary, more and more case reports and studies on a larger population of patients are required to accurately understand the natural history and prognosis of this phenomenon.

\section{Conclusion}

Clinicians and radiologists should be aware of this rare complication of intravitreal $\mathrm{SiO}$ tamponade. Eliciting the history of intraocular $\mathrm{SiO}$ tamponade and identifying the key imaging features on ultrasonography of the orbit, CT of the head, and MRI of the brain will help in avoiding unnecessary interventions or delays in the management of the patients from misdiagnosing it as haemorrhage or tumour. The long-term effects of intracranial $\mathrm{SiO}$ are yet to be elucidated. From what we have learned from our case, we propose that $\mathrm{SiO}$ may have some toxic effects on the CNS leading to encephalopathy, which only time will tell as more cases get revealed. 


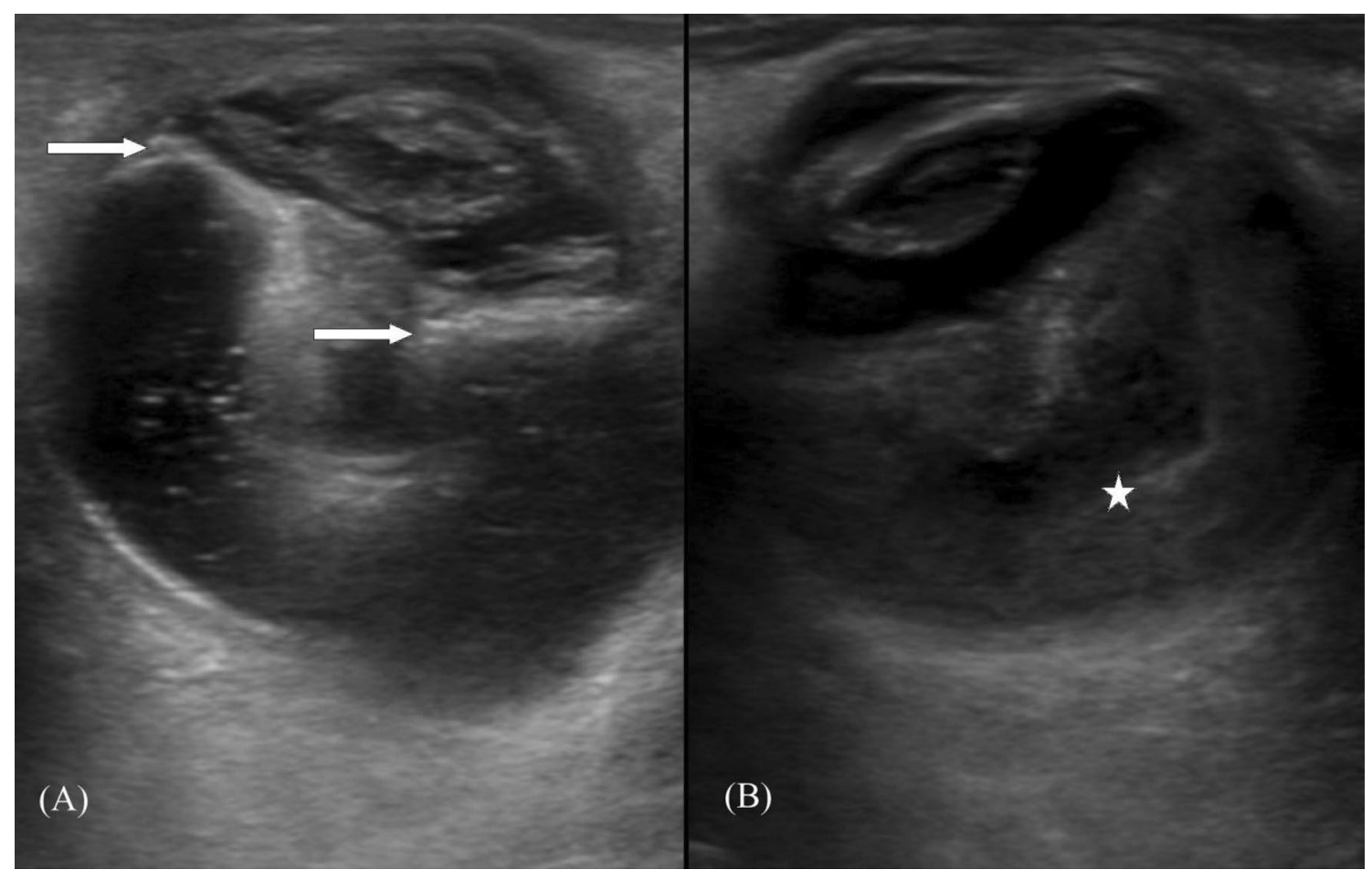

Fig. 6 A Transverse sonogram of the right orbit through the lens shows silicone oil globules as anechoic areas with hyperreflective dots within the globules (due to emulsified silicone droplets), and echogenic arcs (arrows) at the interface of the globules and the vitreous chamber. Lens shows cataractous changes. B Transverse sonogram of the left orbit through the lens shows evidence of chronic total retinal detachment (asterisk) obliterating the vitreous chamber and cataractous changes in the lens

\section{Abbreviation}

PDR: Proliferative diabetic retinopathy; IMSC: Immature senile cataract; RD: Retinal detachment; SiO: Silicone oil; NCCT: Non-contrast computed tomography; CVA: Cerebrovascular accident; HU: Hounsfield unit; MRI: Magnetic resonance imaging; CSF: Cerebrospinal fluid; CT: Computed tomography; CNS: Central nervous system; $\mathrm{SF}_{6}$ : Sulphur hexafluoride; $\mathrm{C}_{3} \mathrm{~F}_{8}$ : Perfluoropropane; IVH: Intraventricular haemorrhage; $\mathrm{VH}$ : Vitreous haemorrhage; MRI: Magnetic resonance imaging; T1Wl:T1-weighted imaging; T2Wl:T2-weighted imaging; GRE: Gradient recalled echo; DWI: Diffusion-weighted imaging; SWI: Susceptibility-weighted imaging; IPH: Intraparenchymal haemorrhage; FLAIR: Fluid-attenuated inversion recovery.

\section{Acknowledgements}

We are thankful to Dr. N.KThulaseedharan, Professor and Head of Dept. of General Medicine, and Dr. Prasoon Sebastian, a Junior resident, for providing the clinical details of the patient.

\section{Authors' contributions}

JP conceived the presented idea and wrote the final manuscript. NN reviewed the literature and wrote the initial manuscript. DE helped in supervising the image selection. FRA helped in editing the manuscript. SM and AP supervised the findings of the work. All authors revised and approved the final version of the manuscript. All authors read and approved the final manuscript.

\section{Funding}

None.

\section{Availability of data and materials}

Data and materials are available to be deposited in publicly available repositories.

\section{Declarations}

Ethical approval and consent to participate

Ethical approval is not required at our institution to publish an anonymous case report.

\section{Consent for publication}

Written informed consent was obtained from the patient.

\section{Competing interests}

The authors declare that they have no competing interests.

\section{Author details}

'Department of Radiodiagnosis, Government Medical College, Kozhikode, Kerala 673008, India. ${ }^{2}$ QQRAA Hospital, Vazhakkad, Kozhikode, Kerala, India.

Received: 7 October 2021 Accepted: 9 January 2022

Published online: 27 January 2022

\section{References}

1. Wilkinson CP, Hinton DR, Sadda SVR, Wiedemann P, Schachat AP. RyansRetina E-Book: 3 Volume Set. Elsevier Health Sciences; 2017. 1821-47. ISBN: 978-0-323-40198-2

2. Federman JL, Schubert HD (1988) Complications associated with the use of silicone oil in 150 eyes after retina-vitreous surgery. Ophthalmology 95(7):870-876 (PMID: 3174036) 
3. Boren RA, Cloy CD, Gupta AS, Dewan VN, Hogan RN (2016) Retrolaminar migration of intraocular silicone oil. J Neuro-Ophthalmol Off J N Am Neuro-Ophthalmol Soc 36(4):439-447 (PMID: 27636746)

4. Papp A, Tóth J, Kerényi T, Jäckel M, Süveges I (2004) Silicone oil in the subarachnoidal space-a possible route to the brain? Pathol Res Pract 200(3):247-252 (PMID: 15200277)

5. Budde M, Cursiefen C, Holbach LM, Naumann GO (2001) Silicone oilassociated optic nerve degeneration. Am J Ophthalmol 131(3):392-394 (PMID: 11239883)

6. Potts MB, Wu AC, Rusinak DJ, Kesavabhotla K, Jahromi BS (2018) Seeing floaters: a case report and literature review of intraventricular migration of silicone oil tamponade material for retinal detachment. World Neurosurg 115:201-205 (PMID: 29678701)

7. Carneiro F, Vaz ND (2019) Prone CT for diagnosis of silicone oil intraventricular migration after intraocular tamponade. Radiol Case Rep 14(7):864-867 (PMID: 31193056)

8. Tang R, Carter K, Woodward J, Pardo G, Sabates F, Heravi MH et al (1994) Silicone and blindness: computed tomography imaging. J NeuroOphthalmol Off J N Am Neuro-Ophthalmol Soc 14(3):155-156 (PMID: 7804418

\section{Publisher's Note}

Springer Nature remains neutral with regard to jurisdictional claims in published maps and institutional affiliations.

\section{Submit your manuscript to a SpringerOpen ${ }^{\odot}$ journal and benefit from:}

- Convenient online submission

- Rigorous peer review

- Open access: articles freely available online

- High visibility within the field

- Retaining the copyright to your article

Submit your next manuscript at $\boldsymbol{\nabla}$ springeropen.com 